\title{
Human bronchial fibroblasts exhibit a mesenchymal stem cell phenotype and multilineage differentiating potentialities
}

\author{
Federica Sabatini ${ }^{1,3}$, Loredana Petecchia ${ }^{1}$, Manuela Tavian $^{2}$, Vanina Jodon de Villeroché ${ }^{3}$, \\ Giovanni A Rossi ${ }^{1}$ and Danièle Brouty-Boyé ${ }^{3}$ \\ ${ }^{1}$ Pulmonary Disease Unit, G. Gaslini Institute, Genoa, Italy; ${ }^{2}$ INSERM U506, IFR André Lwoff, Université Paris \\ XI, Villejuif, France and ${ }^{3}$ INSERM U602, IFR André Lwoff, Université Paris XI, Villejuif, France
}

\begin{abstract}
Mesenchymal stem cells (MSCs) are multipotent cells able to differentiate along different pathways including chondrogenic, osteogenic and adipogenic lineages. MSCs with a fibroblast-like morphology have been identified in human fetal lung. However, their frequency and characterization in human adult lung have not been yet evaluated. Therefore, we analyzed the mesenchymal phenotype and differentiation ability of cultured human adult bronchial fibroblast-like cells $(\mathrm{Br})$ in comparison with those of mesenchymal cell progenitors isolated from fetal lung (ICIG7) and adult bone marrow (BM212) tissues. Surface immunophenotyping by flow cytometry revealed a similar expression pattern of antigens characteristic of marrow-derived MSCs, including CD34 (-), CD45 (-), CD90/Thy-1 (+), CD73/SH3, SH4 (+), CD105/SH2 (+) and CD166/ALCAM (+) in Br, ICIG7 and BM212 cells. There was one exception, STRO-1 antigen, which was only weakly expressed in Br cells. Analysis of cytoskeleton and matrix composition by immunostaining showed that lung and marrow-derived cells homogeneously expressed vimentin and nestin proteins in intermediate filaments while they were all devoid of epithelial cytokeratins. Additionally, $\alpha$-smooth muscle actin was also present in microfilaments of a low number of cells. All cell types predominantly produced collagen and fibronectin extracellular matrix as evidenced by staining with the monoclonal antibodies to collagen prolyl 4-hydroxylase and fibronectin isoforms containing the extradomain (ED)-A together with ED-B in ICIG7 cells. Br cells similarly to fetal lung and marrow fibroblasts were able to differentiate along the three adipogenic, osteogenic and chondrogenic mesenchymal pathways when cultured under appropriate inducible conditions. Altogether, these data indicate that MSCs are present in human adult lung. They may be actively involved in lung tissue repair under physiological and pathological circumstances.
\end{abstract}

Laboratory Investigation (2005) 85, 962-971. doi:10.1038/labinvest.3700300; published online 30 May 2005

Keywords: human fibroblast; lung tissue; mesenchymal differentiation potential; mesenchymal phenotype; mesenchymal stem cell

It is now well accepted that mesenchymal stem cells (MSCs) can be isolated from some human tissues, including adult bone marrow, peripheral blood, adipose tissue, articular synovium, amniotic fluid and other fetal tissues. ${ }^{1-7}$ These MSCs share common characteristics including adherence to culture plastic, fibroblastic-like morphology and long-term proliferation ex vivo. They are generally defined as devoid of hematopoietic and endothelial markers (CD11c, CD31 and CD45) and express at variable extent several other surface antigens including CD90

Correspondence: Dr D Brouty-Boyé, PhD, Inserm U602, Bâtiment Lavoisier, Secteur jaune Hôpital Paul Brousse, 16 avenue Paul Vaillant-Couturier, 94807 Villejuif cedex, France.

E-mail: brouty@vjf.inserm.fr

Received 9 December 2004; revised 5 April 2005; accepted 14 April 2005; published online 30 May 2005
(Thy-1), SH2 (CD105 or endoglin), SH3 or SH4 (CD73) and STRO-1. ${ }^{1,8}$ However, the most reliable biological criterion used to define MSCs remains their capacity to generate the three types of mesenchymal-derived tissue the bone, cartilage and fat tissue. ${ }^{9}$

The ease of culture expansion and genetic manipulation of MSCs appears as one promising therapeutical approach to repair and correct diseased tissues of mesenchymal origin. Many experimental studies focus on the systemic and sitedirected administration of bone marrow-derived MSCs. The identification of resident MSCs in other adult tissues remains limited. For instance, the presence of MSCs in adult lung has not been yet documented even though lung MSCs might be logically more efficient therapeutically than the genetically modified bone marrow-derived MSCs 
currently under investigation for the treatment of lung diseases. ${ }^{10-12}$

In the present study, we analyzed the mesenchymal phenotype and differentiation properties of fibroblast-like cells isolated from normal bronchial (Br) tissue in comparison with fetal lung and adult bone marrow-derived MSC progenitors. We show that $\mathrm{Br}$ fibroblast-like cells display in vitro a phenotype and multi-differentiation potentialities similar to those of the expanded MSC progeny from fetal lung and adult bone marrow tissue.

\section{Materials and methods}

\section{Bronchial Biopsy Samples}

Bronchial biopsies $(n=3)$ were obtained from surgical specimens from smoker patients undergoing lobectomy for primary lung tumors. Tissues were removed distally to the tumor and found to be histologically free of neoplastic involvement, and to display a normal diploid karyotype. All biopsies were taken after the patients had given their informed consent.

\section{Cell Culture}

$\mathrm{Br}$ cells were isolated by enzymatic digestion as described previously for nasal tissue. ${ }^{13}$ Briefly, after trimming off the bronchi from lung parenchyma and peribronchial vascular structures, tissues were digested for $2 \mathrm{~h}$ at $37^{\circ} \mathrm{C}$ using collagenase $(500 \mathrm{U} / \mathrm{ml})$, hyaluronidase $(30 \mathrm{U} / \mathrm{ml})$ and DNAse $(10 \mathrm{U} / \mathrm{ml})$ from Sigma, St Quentin-Fallavier, France, in Dulbecco'smodified Eagle medium (DMEM; GIBCO-BRL, Cergy-Pontoise, France). Cells were then collected by centrifugation, washed twice and resuspended in high-glucose medium supplemented with 10\% fetal calf serum (FCS, Gibco). All cells were plated in T25 $\mathrm{cm}^{2}$ culture flasks (ATGC Biotechnologie, Noisyle-Grand, France) in growth medium with a medium change twice a week until cells reach confluency. To maintain MSC differentiating potentialities, cells were serially subcultured at low cell density (approximately 10 cells $/ \mathrm{cm}^{2}$ ). Human fetal lung fibroblasts ICIG7, were generously provided by Dr Azzarone, Villejuif, France and isolated from a human second-trimester fetus as described. ${ }^{14} \mathrm{Hu}-$ man bone marrow fibroblasts BM212, were isolated from a patient undergoing hip replacement surgery as previously described. ${ }^{15}$ Analyses were performed at different passage (p) levels (from p4 to p10 for Br and BM212 cells and from p15 to p20 for ICIG7 cells).

\section{Cell Immunophenotyping}

The various monoclonal antibodies (MAbs) against surface antigens used, were as follows: fluorescein isothiocyanate (FITC) or phycoerythrin (PE)-conju- gated anti-human CD34, CD45, CD90 (Thy-1), CD73 (SH3 or SH4), CD105 (SH2) and CD166 (activated leukocyte-cell adhesion molecule (ALCAM)) from BD Biosciences, Le Pont de Claix, France; FITCconjugated anti-ASO2 (Thy-1) from Dianova GmbH, Hamburg, Germany; STRO-1 (clone NS1-Ag4-1) from Iowa Hybridoma Bank, Iowa City, USA; FITCconjugate goat anti-mouse Immunoglobulins (IgGs) from Beckman Coulter, Villepinte Roissy CDG, France. Cells were stained according to conventional direct or indirect techniques. ${ }^{16}$ Unspecific staining was determined using isotype-matched nonreactive mouse IgGs (Beckman Coulter). Cells were analyzed on a FACScan ${ }^{\mathrm{TM}}$ (BD Biosciences, Franklin, NJ, USA) using Cell Quest (BD Biosciences) and WinMDI (Scripps Research Institute, La Jolla, CA, USA) software. At least 5000 events were analyzed in each test.

MAbs used against cytoskeletal and extracellular matrix elements were as follows: anti-human vimentin, anti-human pan-cytokeratin (CK) and anti-human $\alpha$-smooth muscle actin (ASMA) from Sigma-Aldrich, St Quentin-Fallavier, France; antihuman prolyl 4-hydroxylase (clone 5B5) from DAKO, Trappes, France; anti-human nestin from R\&D Systems, Lille, France; anti-human extradomain (ED)-A fibronectin (clone IST-9) and ED-B fibronectin (clone BC-1), a gift from Dr L Zardi, I.S.T., Genoa, Italy. Cells were seeded onto eightchamber Permanox Lab-Tek (Nalg Nunc, ATGC Biotechnologie, Marne-Lavallée, France), fixed with methanol for $5 \mathrm{~min}$ at $-20^{\circ} \mathrm{C}$, and processed as previously described. ${ }^{17}$ Fluorescent staining was observed on a DMR/HCS fluorescence microscope (Leica, Reuil-Malmaison, France) and images were acquired using Leica Lida software. The percentage of positive cells was determined on a total of 100 cells distributed in four different fields.

\section{Adipogenic, Chondrogenic and Osteogenic Differentiation Assays}

The potential of $\mathrm{Br}$ cells to differentiate into adipogenic, osteogenic and chondrogenic lineages was assayed at different passage levels. Cells were seeded in culture plates at $2.5 \times 10^{4} \mathrm{cells} / \mathrm{cm}^{2}$ in standard growth medium until confluence. Cells were then stimulated under appropriate inducible conditions. Unstimulated cells and cells treated with identical amounts of diluents were used as controls.

For adipogenic differentiation, ${ }^{7}$ cells were stimulated with growth medium supplemented with $10^{-6} \mathrm{M}$ dexamethasone, $100 \mu \mathrm{g} / \mathrm{ml}$ 3-isobutyl-1methylxanthine, $50 \mu \mathrm{M}$ indomethacin and $10 \mu \mathrm{g} / \mathrm{ml}$ insulin (all from Sigma-Aldrich) for 3 weeks with a medium change twice a week. Cells were then rinsed twice with phosphate-buffered saline (PBS), fixed with $10 \%$ formalin for $10 \mathrm{~min}$, washed with distilled water, rinsed in 60\% isopropanol and 
covered with a $0.3 \%$ oil red O solution (SigmaAldrich) in $60 \%$ isopropanol. After $10 \mathrm{~min}$, cultures were briefly rinsed in 60\% isopropanol and thoroughly in distilled water and let dried at room temperature.

For chondrogenic differentiation, a modified technique described by Muraglia et al was used. ${ }^{17}$ Briefly, cells were stimulated in growth medium supplemented with $1 \mathrm{ng} / \mathrm{ml}$ recombinant human transforming growth factor-beta1 (TGF- $\beta 1$ ) from R\&D systems, for 6 days. Cells were then rinsed twice with PBS, fixed with $10 \%$ formalin for $10 \mathrm{~min}$ at room temperature and washed with distilled water. For immunohistochemical analysis, cells were rehydrated in PBS and incubated in 5\% hydrogen peroxide for $10 \mathrm{~min}$. Nonspecific antibody binding was blocked by incubation with FCS diluted 1:20 in PBS containing 0.2\% Triton X-100 (Sigma-Aldrich) for $30 \mathrm{~min}$. Cells were then incubated with mouse anti-human type II collagen MAb (Chemicon International, Inc., Euromedex, Souffelweyersheim, France) diluted 1:5 in 0.5\% FCS in PBS for $30 \mathrm{~min}$. Slides were further incubated with biotinylated goat anti-mouse IgGs (Beckman Coulter) diluted 1:1000 in 0.5\% FCS in PBS for $30 \mathrm{~min}$. The antibody-biotin conjugates were detected with streptavidin-biotin-horseradish peroxidase complex (Beckman Coulter) applied for $30 \mathrm{~min}$ using diaminobenzidine (Sigma) as substrate. All incubations were carried out at room temperature. Nuclei were counterstained with hematoxylin. Negative controls were unstimulated and TGF- $\beta 1$-treated cells incubated with normal mouse IgGs instead of the primary antibody.

For osteogenic differentiation, ${ }^{3}$ cells were stimulated every 3-4 days in growth medium supplemented with $10^{-7} \mathrm{M}$ dexamethasone, $10 \mathrm{mM} \beta$ glycerophosphate disodium and $50 \mu \mathrm{g} / \mathrm{ml}$ ascorbic acid (all from Sigma). After 4 weeks, cells were rinsed twice with PBS, fixed with formalin for $10 \mathrm{~min}$, and washed with distilled water. To stain calcium deposits, cells were covered with a $2 \%$ aqueous solution of alizarin red S (Sigma), $\mathrm{pH} 4.2$, for $3 \mathrm{~min}$. Cultures were then washed thoroughly with distilled water and let dried at room temperature.

\section{Gene Expression}

Reverse-transcriptase polymerase chain reaction (RT-PCR) was used to determine the expression of peroximose proliferator-activated receptor $\gamma 2$ (PPAR $\gamma 2$ ), osteopontin, type II collagen, and Sox-9, in cultures stimulated towards differentiation. Target genes were amplified by PCR with the following primers: human PPAR 2 , 5' -AGGAGCA-GAGCAAA GAGGTG-3' and $5^{\prime}$-AGGACTCAGGGT-GGTTCAGC$3^{\prime}$; human osteopontin, 5'-GCTAAACCCTGACC CATCTC-3' and $5^{\prime}$-ATAACTGTCCTTCC-CACGGC$3^{\prime}$; human Sox-9, 5'-AGCAGCGAAATCAACGA
GAA-3 ${ }^{\prime}$ and $5^{\prime}$-CAAAGTCCAAACAG-CAGAGA-3'; human collagen II, 5'-CTGCTCGTCGCCGCTGTCC TT-3' and 5'-GGGTCCCAGGTT-CTCCATC- ${ }^{\prime}$; the house-keeping gene human $\beta$-actin, 5'TGACGGG GTCACCCACACTGTGCCCAT-CTA- $3^{\prime}$ and $5^{\prime}$-CTAG AAGCATTTGCGGTGGACGATGGAGGG-3' .

\section{Results}

Immunophenotype of Human Adult Br vs Fetal Lung and Adult Bone Marrow-Derived Fibroblasts

Flow cytometry analysis revealed that $\mathrm{Br}$ cells homogeneously expressed ASO2 (>90\%), a CD90 antigen epitope specifically exposed on the surface of fibroblasts ${ }^{18}$ (Figure 1). In addition, they also strongly expressed the mesenchymal stem/progenitor cell surface antigens CD73, CD105 and CD166 while the expression of STRO-1 was weak. No expression of the endothelial and blood cell antigens CD31, CD34 and CD45 was monitored (not shown). The immunophenotypic profile of $\mathrm{Br}$ cells was similar to that of fetal lung IGIG7 and bone marrow BM212 fibroblasts except that STRO-1 was expressed. STRO-1 has been reported to identify human clonogenic bone marrow stromal progenitors able to give rise to cells with the phenotype of fibroblasts, adipocytes, osteocytes and smooth muscle cells. ${ }^{19}$

No subpopulations could be distinguished in any cell types on the basis of cell morphology and cellular size and granularity by flow cytometry (Figure 2a). Moreover, all cells homogeneously expressed CD90 (Figure 2b), a surface antigen reported to identify different fibroblast subsets with functional diversity. ${ }^{20,21}$

Further evaluation of cystoskeletal and extracellular matrix elements by immunofluorescent staining showed that only a few $(<6 \%)$ cells stained positively for the myofibroblast marker ASMA in microfilaments (Figure 3a). Such a low numbers of ASMA $(+)$ cells were similarly detected in ICIG7 and BM212 fibroblasts. Moreover, nearly all Br cells expressed vimentin, nestin but not epithelial panCK in intermediate filaments. Additionally, a homogeneous staining for collagen prolyl 4-hydroxylase and ED-A fibronectin isoform was observed (Figure 3b). A similar expression pattern was monitored in the two other ICIG7 and BM212 fibroblasts, except that the ED-B fibronectin isoform was abundantly present in fetal lung ICIG7 cells. Table 1 summarizes the results.

\section{Differentiation of Adult Br vs Fetal Lung and Adult Bone Marrow Fibroblasts along Adipogenic, Chondrogenic and Osteogenic Lineages}

A marked adipogenesis was induced in the three types of fibroblasts under inducible culture conditions as revealed by oil red $\mathrm{O}$ staining (Figure 4). 

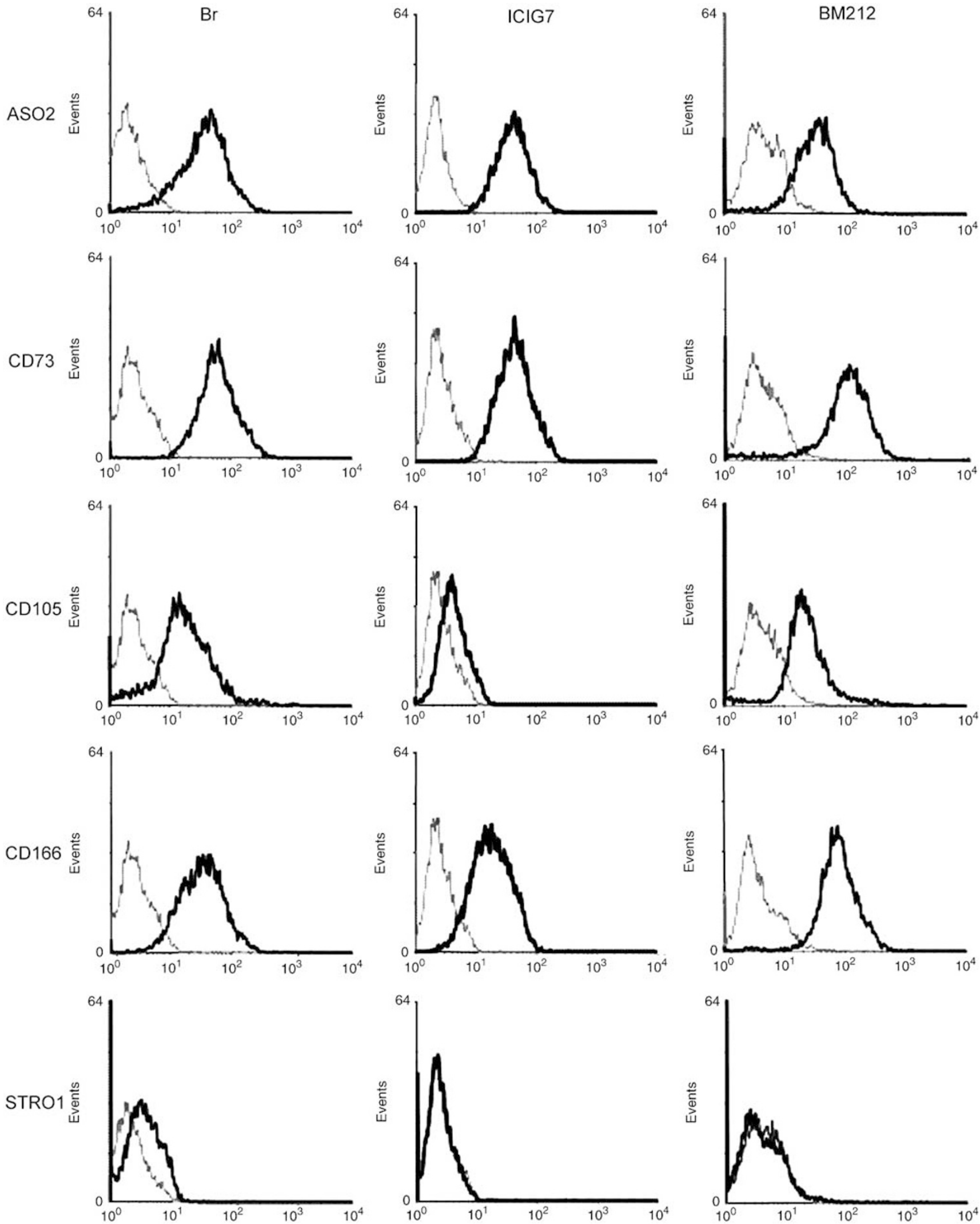

Figure 1 Expression of mesenchymal stem cell/progenitor markers in adult Br compared with fetal lung and bone marrow-derived fibroblasts. Cells were stained with indicated antibodies and analyzed by flow cytometry. Depicted is the cell reactivity to MAbs (solid lines) and irrelevant isotype IgGs (broken lines). Note: the weak expression of STRO-1 antigen in Br fibroblasts and its absence in the other two. Data are representative of more than three independent experiments. 

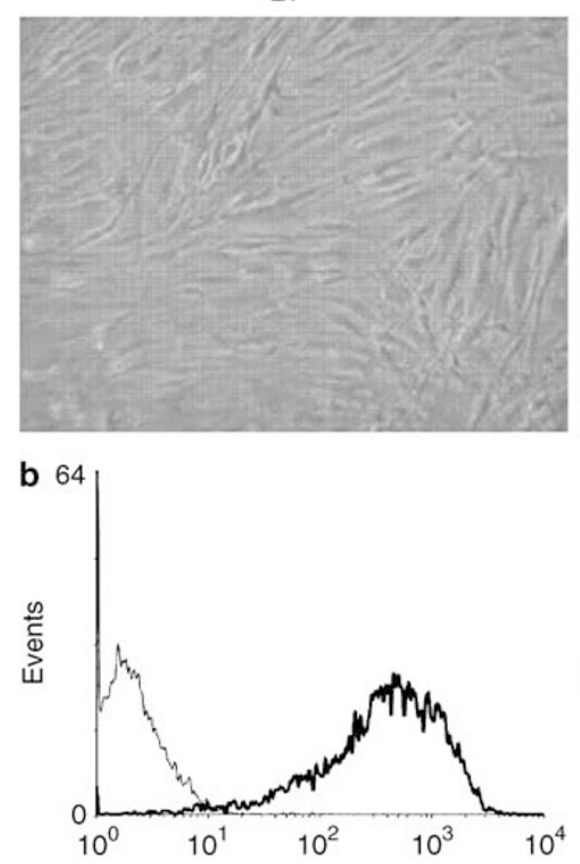

ICIG7
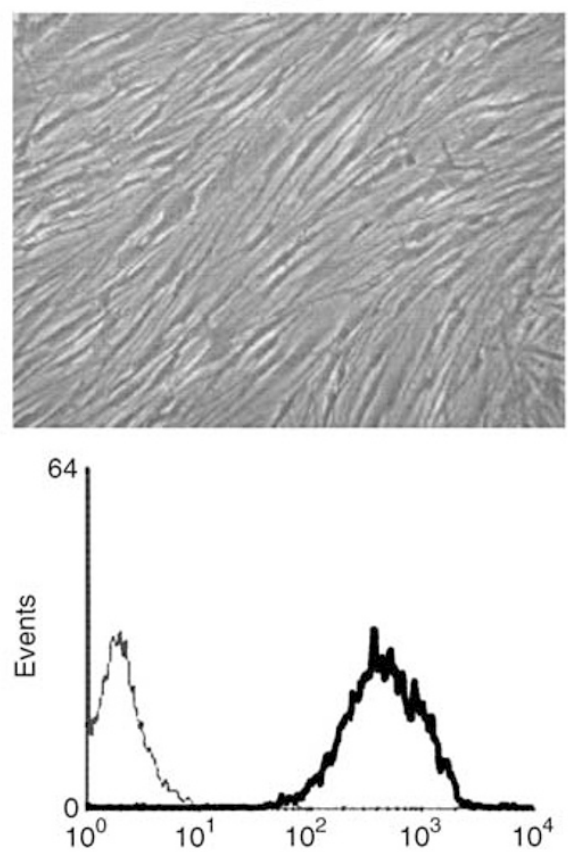

BM212
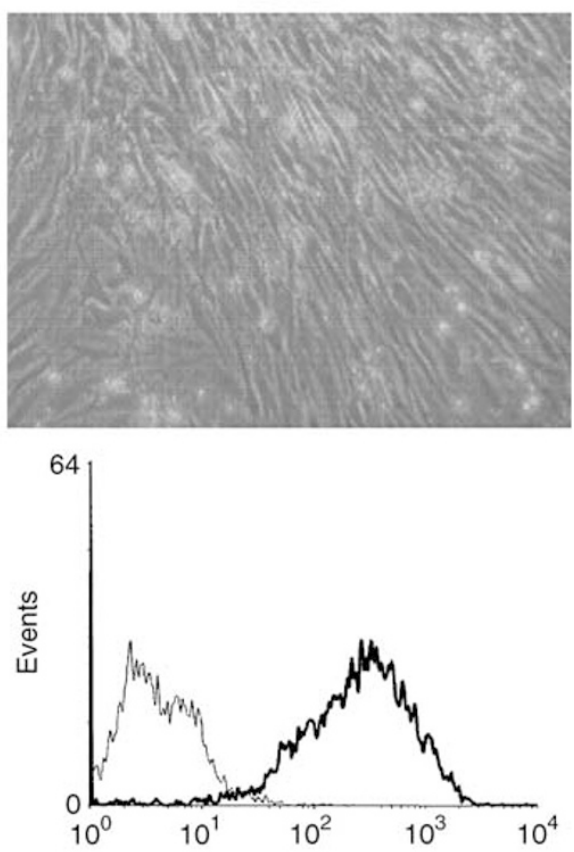

Figure 2 Morphology and CD90 expression in adult Br vs fetal lung and adult bone marrow-derived fibroblasts. Top panels (a) shows the morphology of confluent cultures viewed under inverted phase-contrast microscope (original magnification $\times 100)$. Bottom panels $(\mathbf{b})$ show CD90 expression (solid lines) by flow cytometry. Controls were performed with an irrelevant isotype IgGs (broken lines). Note: the homogeneous morphology and CD90 expression in all cases. Data are representative of at least three independent experiments.

However, a consistent $(15 \%)$ percentage of cells were also stained in untreated $\mathrm{Br}$ and ICIG7 lungderived controls while absent from untreated bone marrow BM212 controls. It should be noted that in BM212 cultures devoid of basal lipid-containing cells, stimulation by adipogenic medium induced within 1 week changes in cell morphology as well as in the content of lipid droplets that progressively increased in number, coalesced and eventually filled the whole cells by week 3 .

Br cells, similarly to fetal lung ICIG7 and adult bone marrow BM212 fibroblasts underwent chondrogenic differentiation when treated with $1 \mathrm{ng} / \mathrm{ml}$ of TGF- $\beta 1$ for 1 week as evidenced by the presence of extracellular cartilage type II collagen immunostaining (Figure 5). This process was not associated with an increase in ASMA expression (data not shown), indicating a chondrocyte-like rather than myofibroblast phenotype induced by TGF- $\beta 1$ treatment. Untreated control cultures were all negative.

Lastly, all three cell types underwent morphologic changes and formed cell piling up under osteogenic culture conditions (Figure 6). Moreover, abundant amounts of calcium deposits were detected by alizarin red staining consistent with bone formation. Untreated control cultures were all negative.
The mesenchymal differentiation potential of $\mathrm{Br}$ cells was consistent with the upregulation in the expression of other lineage-specific genes such as PPAR 2, Sox-9 and type II collagen, and osteopontin for adipogenic, chondrogenic and osteogenic activity, respectively, as shown by RT-PCR (Figure 7).

\section{Discussion}

The present study shows that human morphologically fibroblast-like cells isolated from adult $\mathrm{Br}$ tissue expressed most of the surface antigens characteristic of MSCs isolated from other adult and fetal tissue sources. ${ }^{1,5,7}$ The surface antigenic profile of $\mathrm{Br}$ cells appeared similar to that of fetal lung and adult bone marrow-derived MSC fibroblastic progenitors except that they weakly expressed STRO-1 antigen whereas the other two did not. STRO-1 is a not yet defined cell surface antigen present in a minor multipotent subpopulation of adult human bone marrow stromal cells growing in long-term culture, the expression of which declining with increasing culture times. ${ }^{8}$ As MSC populations appear heterogeneous between species and within cultures, variable expression of some of

Figure 3 Expression of various cytoskeletal and extracellular matrix proteins in adult $\mathrm{Br} v s$ fetal lung and adult bone marrow-derived fibroblasts. Cells were stained with indicated antibodies and viewed under fluorescent microscope. (a) Cystoskeletal proteins in Br, ICIG7 and BM212 cells; (b) collagen and fibronectin expression (original magnification $\times 200$ ). Note: the presence of ED-B fibronectin expression in fetal lung ICIG7 fibroblasts. Data are representative of at least three independent experiments. 
a

$\alpha$-smooth muscle actin
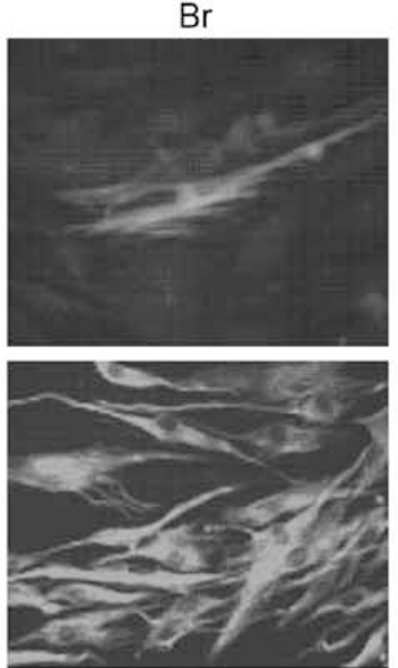

Vimenti

Pan-cytokeratin

b

Collagen

prolyl 4-hydroxylase
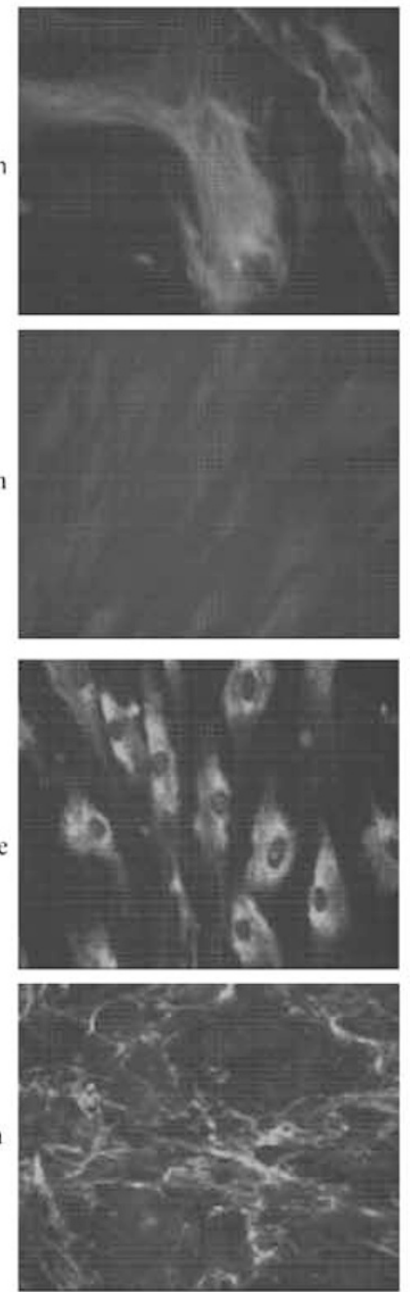

ED-A fibronectin

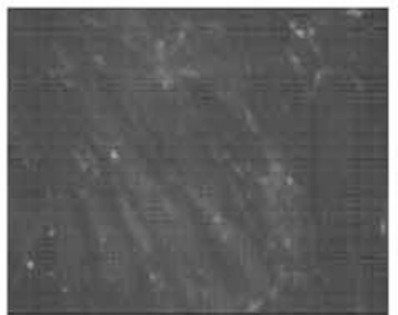

ICIG7
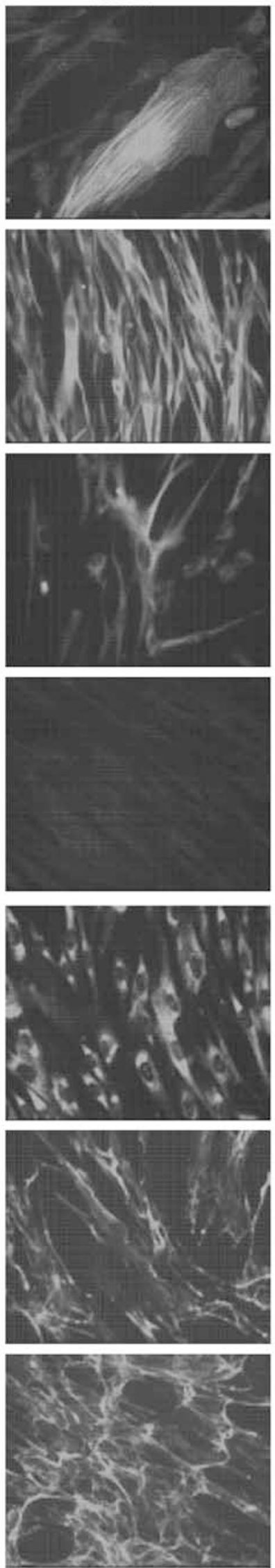

BM212
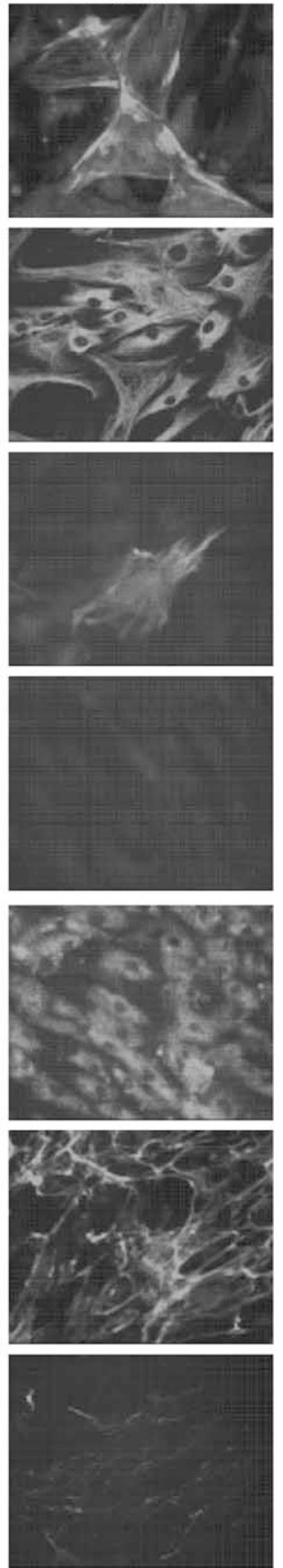
Table 1 Summary of the phenotypic characterization of human fibroblasts isolated from adult and fetal lung and adult bone marrow tissue

\begin{tabular}{|c|c|c|c|}
\hline Antigen & Br cells & Fetal lung ICIG7 cells & Bone marrow BM212 cells \\
\hline \multicolumn{4}{|l|}{ Cell surface molecules } \\
\hline ASO2 & + & + & + \\
\hline CD34 & - & - & - \\
\hline CD45 & - & - & - \\
\hline CD90 & + & + & + \\
\hline CD73 & + & + & + \\
\hline CD105 & + & + & + \\
\hline CD166 & + & + & + \\
\hline STRO-1 & + & - & - \\
\hline \multicolumn{4}{|c|}{ Cytoskeleton and extracellular matrix proteins } \\
\hline ASMA & $+(\leq 6 \%)$ & $+(\leq 1 \%)$ & $+(\leq 3 \%)$ \\
\hline ED-A fibronectin & + & + & + \\
\hline ED-B fibronectin & - & + & - \\
\hline Nestin & $+(100 \%)$ & $+(100 \%)$ & $+(100 \%)$ \\
\hline PanCK & - & - & - \\
\hline Prolyl 4-hydroxylase & $+(100 \%)$ & $+(100 \%)$ & $+(100 \%)$ \\
\hline Vimentin & $+(100 \%)$ & $+(100 \%)$ & $+(100 \%)$ \\
\hline
\end{tabular}

Analyses were performed on Br cells from three different isolates between p3 and p7, bone marrow cells between p3 and p7, and fetal lung cells between p15 and p20. Positive (+) or negative $(-)$ indicate the presence or absence of antigen expression, respectively. Numbers in parenthesis indicate the percentage $(\%)$ of positive cells for the indicated antigen as monitored under fluorescent microscope.
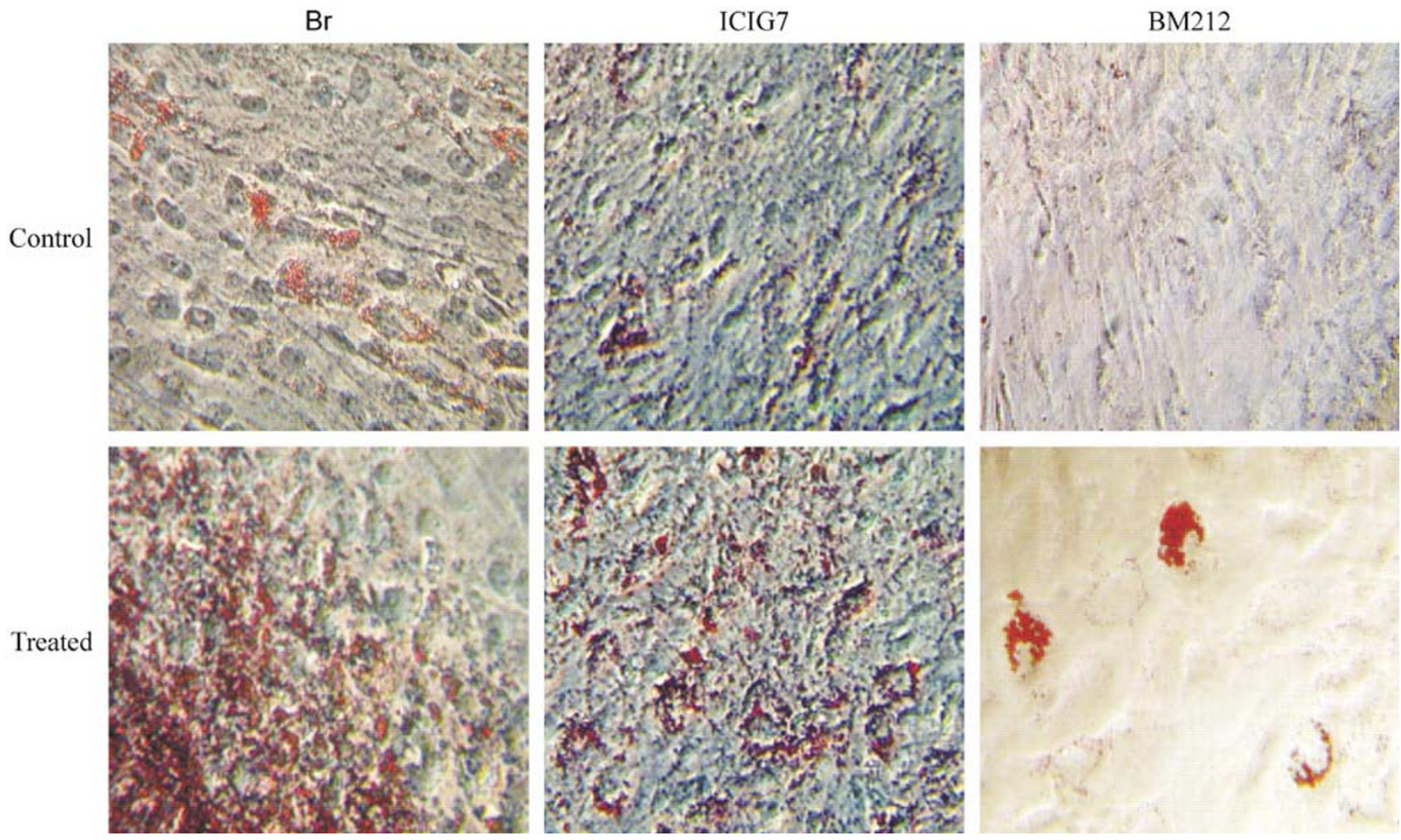

Figure 4 Adipogenic differentiation in adult $\mathrm{Br}$ vs fetal lung and adult bone marrow-derived fibroblasts. Cells were cultured in adipogenic medium (treated) or left untreated (control) for 3 weeks. Accumulation of lipid droplets within the cells was revealed by staining with oil red $\mathrm{O}$ and viewed under inverted phase-contrast microscope (original magnification $\times 200$ ). Note: the basal staining in a few cells from the two untreated cultures from lung origin. Data are representative of three independent experiments.

the mesenchymal markers is commonly reported. It may be due to differences in cell isolation processing, culture conditions and tissue of origin as well.

Besides consensual MSC markers, $\mathrm{Br}$ cells predominantly expressed other antigens that charac- terize ordinary adult tissue fibroblasts, including CD90, vimentin, collagen prolyl 4-hydroxylase, and fibronectin. They also expressed nestin protein in intermediate filaments. Nestin originally described as a predominant neuronal stem/progenitor cell 

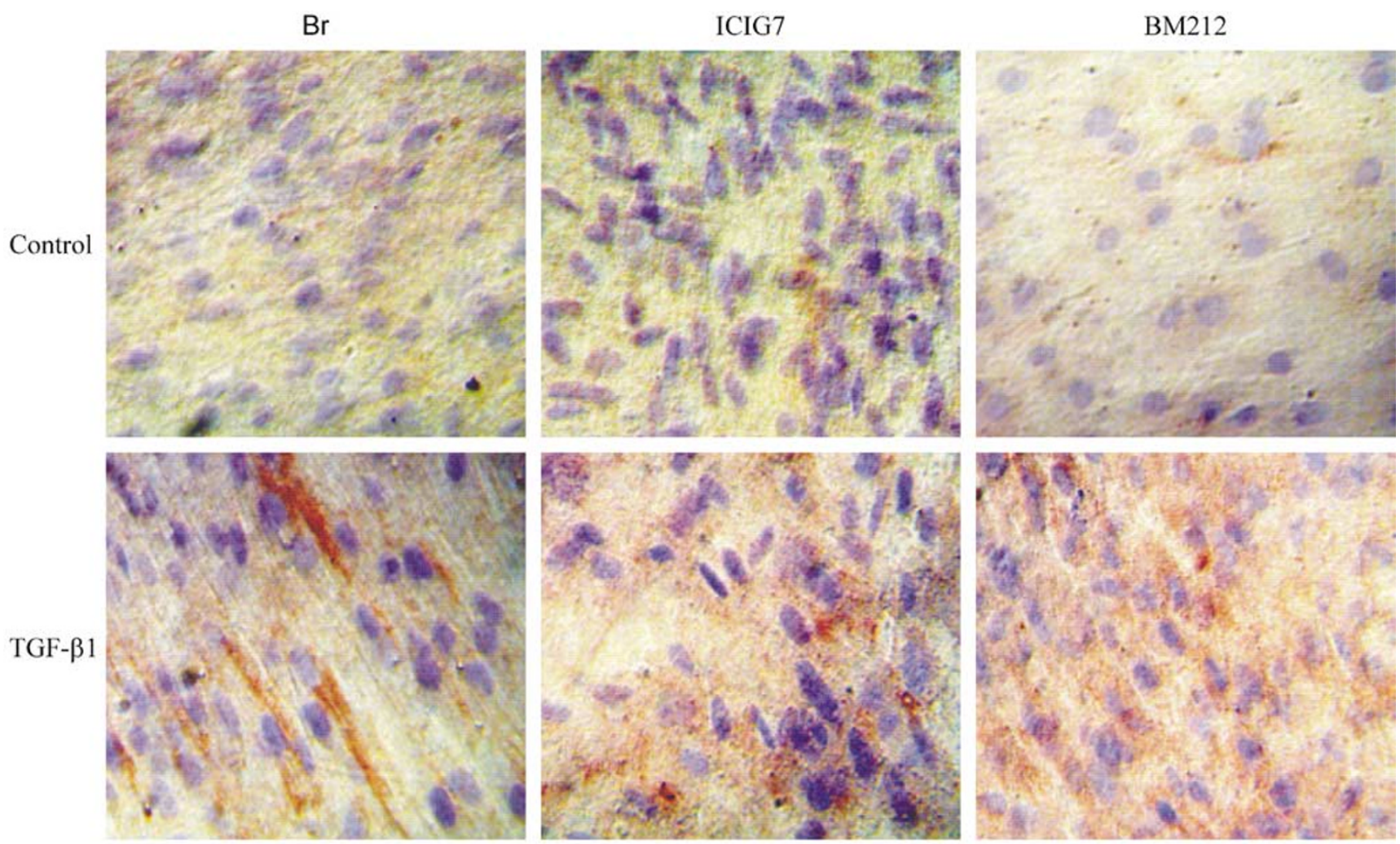

Figure 5 Chondrogenic differentiation in adult $\mathrm{Br}$ vs fetal lung and adult bone marrow-derived fibroblasts. Cells were cultured in the presence of TGF- $\beta 1$ or left untreated (control) for 1 week. Chondrogenic differentiation was revealed by immunohistochemical staining for type II collagen and viewed under phase-contrast microscope (original magnification $\times 200$ ). Data are representative of three independent experiments.

$\mathrm{Br}$
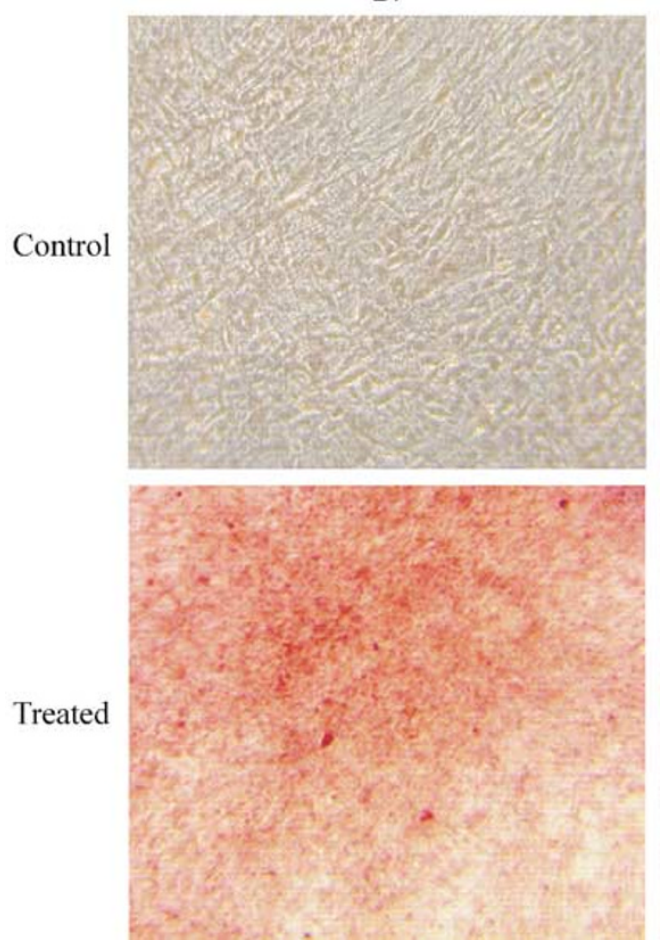

ICIG7
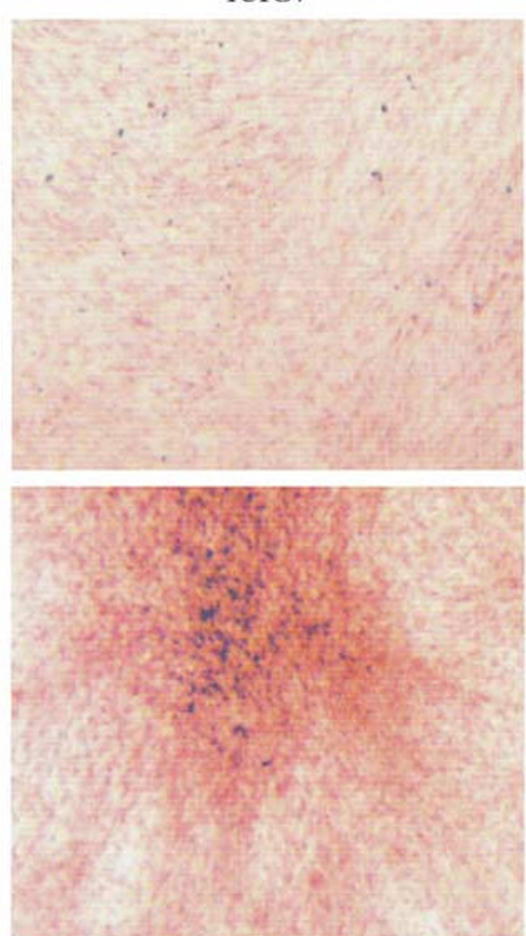

BM212
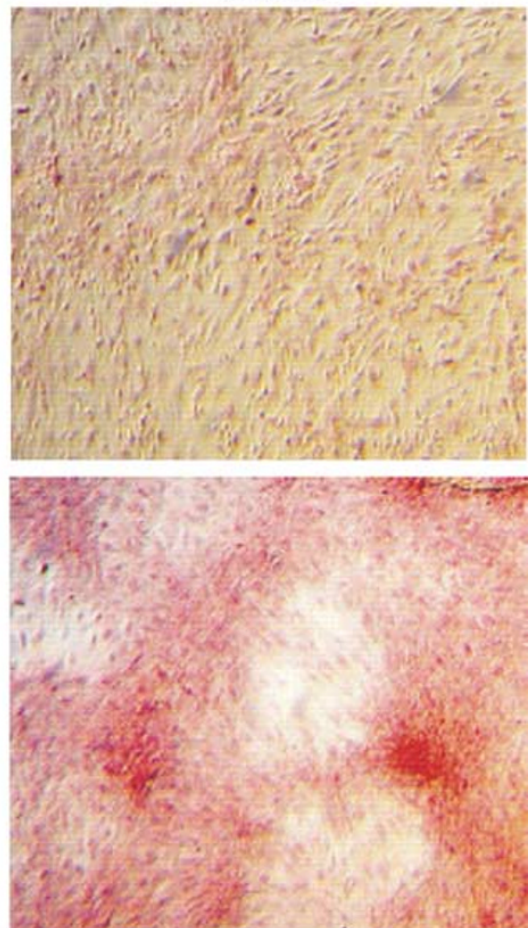

Figure 6 Osteogenic differentiation in adult Br fibroblasts vs fetal lung and adult bone marrow-derived cells. Cells were cultured in osteogenic medium (treated) or left untreated (control) for 4 weeks. Calcium deposits were revealed by staining with alizarin red $S$ and viewed under phase-contrast microscope (original magnification $\times 100$ ). Data are representative of three independent experiments. 


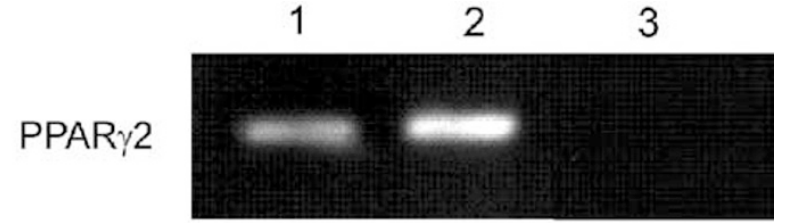

$\beta$-actin

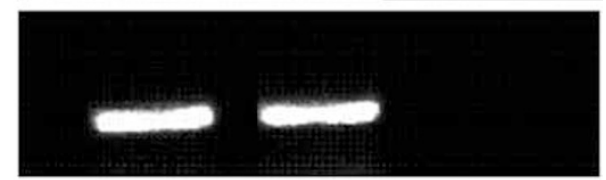

Sox-9

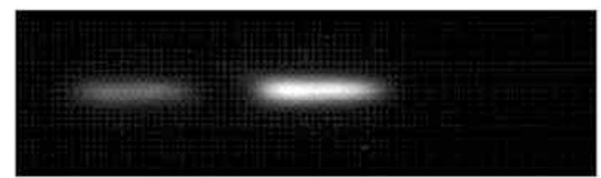

Col2

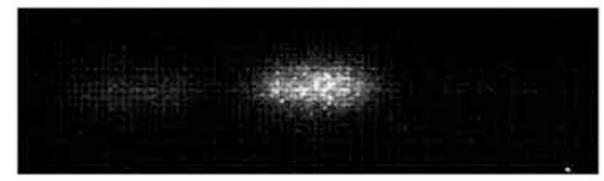

$\beta$-actin

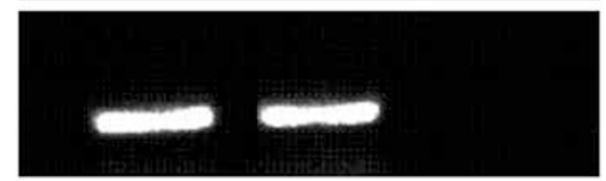

Osteopontin

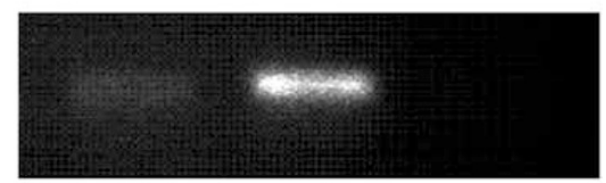

$\beta$-actin

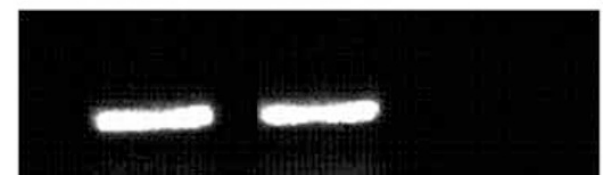

Figure 7 Upregulation of specific adipogenic, chondrogenic and osteogenic markers in Br fibroblasts under inductive culture conditions. Untreated cells (lane 1) and cells under each appropriate differentiation treatment (lane 2) were collected and processed for RT-PCR to analyze the expression of PPAR $\gamma 2$ for adipogenic activity, Sox-9 and type II collagen (Col2) for chondrogenic activity, and osteopontin for osteogenic activity. (Lane 3) milli-Q water negative control. Note: the upregulation of all differentiation genes after stimulation when compared with the basal expression in unstimulated cells. Data are representative of two independent experiments.

marker ${ }^{22}$ is actually expressed in activated cells from diverse fetal and adult tissues and may relate to a common embryonic cell origin, the neural crest. ${ }^{23}$

Regarding cellular heterogeneity, Br cells similarly to fetal lung and adult bone marrow-derived fibroblasts were morphologically homogeneous and only a few myofibroblasts were present. Moreover, we were unable to distinguish cellular subpopulations on the basis of CD90 (Thy-1) expression. CD90 has been reported by Phipps and co-workers to be heterogeneously expressed in fibroblast isolates from various human tissues, including lung, myometrium and orbit. ${ }^{20,21,24}$ More recently, it successfully helped to discriminate Thy-1 $(+)$ fibroblasts able to give rise to myofibroblasts from Thy-1(-) cells able to generate lipofibroblasts but not myofibroblasts under inducible conditions. ${ }^{25}$ Contrastingly, to these studies, we found that among the homogeneously Thy-1-expressing lung cells, the myofibroblast and lipofibroblast phenotypes were spontaneously present as evidenced by ASMA expression and oil red $\mathrm{O}$ staining, respectively.

The presence of lipofibroblasts that contain cytoskeletal proteins characteristics of myofibroblasts, has been previously described in the pulmonary alveolar interstitium. ${ }^{26}$ They were shown to produce extracellular matrix components and contribute to surfactant synthesis in type II pneumocytes via their lipid release. However, lipofibroblasts can convert into myofibroblasts under specific TGF$\beta 1$ stimulation. ${ }^{27}$ The conversion process is associated with a gradual loss of adipogenic differentiation-related markers and metabolic changes that in turn affects phospholipid surfactant synthesis by type II pneumocytes. ${ }^{28}$ We show that the formation of lipofibroblasts was increased in $\mathrm{Br}$ and fetal lung cultures under inducible adipogenic conditions. Most remarkably, Br fibroblasts were also capable of differentiating along the chondrogenic and osteogenic lineages and may be, thus, considered as closely related to MSCs.

It is most likely that resident MSCs in adult tissues are involved in the maintenance and repair of injured tissues. Preliminary studies have indicated that bone marrow-derived MSCs might be applied therapeutically for the correction of disorders from mesenchymal origin as osteogenesis imperfecta. ${ }^{29}$ Furthermore, it has been shown that marrow stem cells were able to deliver gene therapy to lung epithelium while maintaining long-term transgene expression. ${ }^{10}$ The existence of MSCs in the lung we reported here may offer efficient therapeutical approaches to a variety of lung disorders, through the manipulation of processes involved in airway remodeling that follow acute injury, the correction of acquired or genetically determined mesenchymal cell disorders and, possibly, the delivery of a transgenic protein to other airway structural cells, including Br epithelial cells.

\section{Acknowledgements}

We thank Dr B Azzarone for his helpful advices, and Dr M-C Le Bousse-Kerdilès for providing us human bone marrow cells. This work was supported by grants from the Italian Ministry of Health and the French Association NRB.

\section{References}

1 Pittenger MF, Mackay AM, Beck SC, et al. Multilineage potential of adult human mesenchymal stem cells. Science 1999;284:143-147. 
2 Zvaifler NJ, Marinova-Mutafchieva L, Adams G, et al. Mesenchymal precursor cells in the blood of normal individuals. Arthritis Res 2000;2:477-488.

3 De Bari C, Dell'Accio F, Tylzanowski P, et al. Multipotent mesenchymal progenitor cells from adult human synovial membrane. Arthritis Rheum 2001;44: 1928-1942.

4 in 't Anker PS, Scherjon SA, Kleijburg-van der Keur C, et al. Amniotic fluid as a novel source of mesenchymal stem cells for therapeutic transplantation. Blood 2003;102:1548-1549.

5 Zuk PA, Zhu M, Ashjian P, et al. Human adipose tissue is a source of multipotent stem cells. Mol Biol Cell 2002;13:4279-4295.

6 Campagnoli C, Roberts IA, Kumar S, et al. Identification of mesenchymal stem/progenitor cells in human first-trimester fetal blood, liver, and bone marrow. Blood 2001;98:2396-2402.

7 in 't Anker PS, Noort WA, Scherjon SA, et al. Mesenchymal stem cells in human second-trimester bone marrow, liver, lung, and spleen exhibit a similar immunophenotype but a heterogeneous multilineage differentiation potential. Hematologica 2003;88: 845-852.

8 Simmons PJ, Torok-Storb B. Identification of stromal cell precursors in human bone marrow by a novel monoclonal antibody, STRO-1. Blood 1991;78:55-62.

9 Pittenger MF, Mosca JD, McIntosh KR. Human mesenchymal stem cells: progenitor cells for cartilage, bone, fat and stroma. Curr Top Microbiol Immunol 2000;251:3-11.

10 Grove JE, C Lutzko C, Priller J, et al. Marrow-derived cells as vehicles for delivery of gene therapy to pulmonary epithelium. Am J Respir Cell Mol Biol 2002;27:645-651.

11 Koehler DR, Hitt MM, Hu J. Challenges and strategies for cystic fibrosis lung gene therapy. Mol Ther 2001;4:84-91.

12 Gill DR, Davies LA, Pringle IA, et al. The development of gene therapy for diseases of the lung. Cell Mol Life Sci. 2004;61:355-368.

13 Silvestri M, Sabatini F, Scarso L, et al. Fluticasone propionate downregulates nasal fibroblast functions involved in airway inflammation and remodeling. Int Arch Immunol 2002;128:51-58.

14 Azzarone B, Suarez H, Mingari M, et al. 4F2 monoclonal antibody recognizes a surface antigen on spread human fibroblasts of embryonic but not adult origin. J Cell Biol 1984;98:1133-1137.

15 Brouty-Boyé D, Doucet D, Clay D, et al. Phenotypic diversity in human fibroblasts from myelometaplasic and non-myelometaplasic hematopoietic tissues. Int J Cancer 1998;76:767-773.
16 Brouty-Boyé D, Raux $\mathrm{H}$, Azzarone $\mathrm{B}$, et al. Fetal myofibroblast-like cells isolated from post-radiation fibrosis in human breast cancer. Int J Cancer 1991;47: 697-702.

17 Muraglia A, Cancedda R, Quarto R. Clonal mesenchymal progenitors from human bone marrow differentiate in vitro according to a hierarchical model. J Cell Science 2000;113:1161-1166.

18 Saalbach A, Kraft R, Herrman K, et al. The monoclonal antibody ASO2 recognizes a protein on human fibroblasts being highly homologous to Thy-1. Arch Dermatol 1998;290:360-366.

19 Gronthos S, Graves SE, Ohta S, et al. The STRO-1+ fraction of adult human bone marrow contains osteogenic precursors. Blood 1994;84:4164-4173.

20 Derdak S, Penney DP, Keng P, et al. Differential collagen and fibronectin production by Thy-1+ and Thy-1- lung fibroblast subpopulations. Am J Physiol 1992;263:L283-L290.

21 Koumas L, King AE, Critchley HO, et al. Existence of functionally distinct Thy $1+$ and Thy-1- human female reproductive tract fibroblasts. Am J Pathol 2001;159:925-935.

22 Messam CA, Hou J, Major EO. Coexpression of nestin in neural and glial cells in the developing human CNS defined by a huma-specific anti-nestin antibody. Exp Neurol 2000;161:585-596.

23 Vescosi AL, Galli R, Gritti A. The neural stem cells and their transdifferentiation capacity. Biomed Pharmacother 2001;55:201-205.

24 Koumas L, Smith TJ, Phipps RP. Fibroblast subsets in the human orbit: Thy-1+ and Thy-1- subpopulations exhibit distinct phenotypes. Eur J Immunol 2002;32: 477-485.

25 Koumas L, Smith TJ, Feldon S, et al. Thy-1 expression in human fibroblast subsets defines myofibroblastic or lipofibroblastic phenotypes. Am J Pathol 2003;163: 1291-1300.

26 McGowan SE, Torday JS. The pulmonary lipofibroblast (lipid interstitial cell) and its contribution to alveolar development. Annu Rev Physiol 1997;59:43-62.

27 Torday JS, Torres E, Rehan VK. The role of fibroblast transdifferentiation in lung epithelial cell proliferation, differentiation, and repair in vitro. Pediatr Pathol Mol Med 2003;22:189-207.

28 Schultz CJ, Torres E, Londos C, et al. Role of adipocyte differentiation-related protein in surfactant phospholipid synthesis by type II cells. Am J Physiol Lung Cell Mol Physiol 2002;283:L288-L296.

29 Horwitz EM, Prockop DJ, Fitzpatrick LA, et al. Transplantability and therapeutic effects of bone marrow-derived mesenchymal cells in children with osteogenesis imperfecta. Nat Med 1999;5:309-313. 